\title{
COMPORTAMENTO DE CLONES DE PENNISETUM SUBMETIDOS A PERÍODOS DE RESTRIÇÃO HÍDRICA CONTROLADA ${ }^{1}$
}

\author{
RESPONSE OF PENNISETUM CLONS TO PERIODS OF CONTROLLED \\ HIDRIC RESTRICTION
}

\author{
Santos, M.C.S. ${ }^{2 A}$, Lira, M.A. ${ }^{2 B}$, Tabosa, J.N. ${ }^{2 C}$, Mello, A.C.L. ${ }^{3 A}$ e Santos, M.V.F. ${ }^{3 *}$ \\ Instituto Agronômico de Pernambuco-IPA. Brasil. ${ }^{A}$ carmo@ipa.br; ${ }^{B}$ mariolira@terra.com.br; ${ }^{C}$ tabosa@ipa.br \\ ${ }^{3}$ Departamento de Zootecnia. Universidade Federal Rural de Pernambuco.UFRPE.Brasil.Amello@dz.ufrpe.br; \\ *mercia@dz.ufrpe.br
}

\section{Palavras chave adicionais}

Capim-elefante. Estresse hídrico. Evapotranspiração. Eficiência de uso de água.

\section{RESUMO}

O experimento foi desenvolvido em casa de vegetação no Instituto Agronômico de Pernambuco e objetivou avaliar clones de Pennisetum sp. submetidos a diferentes níveis de tempo de supressão da reposição hídrica. $O$ delineamento experimental foi inteiramente casualizado em esquema fatorial, sendo quatro clones de Pennisetum sp. e quatro níveis de estresse hídrico $(0,7$, 14 e 21 dias sem irrigação), com 3 repetições. Foram estimadas a evapotranspiração, a percentagem e a produção de matéria seca (MS), a eficiência de uso de água, a altura média das plantas, o número de perfilhos basais, os sintomas de estresse hídrico nas plantas, o número de plantas rebrotadas e de perfilhos basais após estresse. O maior tempo de supressão da reposição hídrica reduziu mais a evapotranspiração do que a produção de matéria seca, tendo a eficiência de uso de água valores de 449 e $591 \mathrm{~g}$ de água por g de matéria seca nos tratamentos com 21 dias de déficit hídrico e sem estresse hídrico, respectivamente. Sete dias sem irrigação elevaram o teor de MS de $25,11 \%$ para $53,07 \%$ e de $29,25 \%$ para $46,89 \%$, nos clones Australiano e HV 241, respectivamente, não tendo produzido mudanças significativas $(p>0,05)$ nos dois outros clones. Dentre os clones avaliados, o Australiano e o HV

${ }^{1}$ Parte da dissertação apresentada pelo primeiro autor ao Programa de Pós-Graduação em Botânica da Universidade Federal Rural de Pernambuco.

\section{AdDitional KeYWORDS}

Pennisetum purpureum. Water deficit. Evapotranspiration. Water usage efficiency.

241 apresentam maior produção de massa seca quando sujeitas a estresse hídrico moderado.

\section{SUMMARY}

The experiment was conducted in a greenhouse of the Agronomic Institute of Pernambuco, to evaluate Pennisetum sp. clons submitted to different periods of water reposition suppression. The experimental design was completely randomized in a factorial arrangement with four Pennisetum sp. clones and four periods without water reposition $(0,7,14$ and 21 days), with three replicates. Evapotranspiration, dry matter content and production, water use efficiency, average plant height, basal tiller number, water stress symptoms, number of regrowth plants and basal tiller number after the stress were measured. A longer water suppression period affected evapotranspiration more than dry matter production, with water use efficiency being 449 and $591 \mathrm{~g}$ of water per $\mathrm{g}$ of dry matter for treatments with 21 and 0 days of irrigation suppression, respectively. Seven days without irrigation increased dry matter content from 25.11 to 53.07 and from 29.25 to 46.89 for Australiano and HV241 clons, with no significant difference $(p>0.05)$ for the two other clons. Among the evaluated clons, Australiano and HV241 had the highest dry matter production under moderate water deficit. 


\section{INTRODUÇÃO}

Nas regiões semiáridas, o clima é o principal responsável pelas variações estacionais de produção de forragem, definindo assim, a sazonalidade de oferta de forragem em duas estações bem definidas, chuvosa e seca. Deste modo, a disponibilidade de forragem tem sido apontada como o principal fator limitante para o desenvolvimento da pecuária do Nordeste, principalmente no período seco, notadamente nas áreas em que as irregularidades climáticas são mais acentuadas (Santos et al., 1994).

A qualidade e produtividade das pastagens geralmente são influenciadas por fatores ambientais como temperatura, luz e água, além de fatores intrínsecos da própria planta. A temperatura e a disponibilidade hídrica são fatores limitantes ao crescimento e desenvolvimento das plantas, alterando sua estrutura morfológica, fisiológica e bioquímica (Taiz e Zeiger, 1998). Lira et al. (1989) ressaltaram que, principalmente em condições tropicais, a disponibilidade hídrica é o fator climático de maior efeito sobre a produtividade agrícola. Além disto, a ocorrência simultânea de altas temperaturas e baixas umidades relativa do ar proporcionam uma taxa de evapotranspiração potencial mais alta do que a disponibilidade de água (Lehane, 1981).

Por outro lado, evidencia-se que a formação de capineiras constitui uma das alternativas para a redução dos prejuízos causados pela estacionalidade de produção de forragem, sendo o capim-elefante (Pennisetum purpureum Schum.) uma opção de forrageira tropical de alto potencial produtivo, cultivada em toda região intertropical do globo, bem como na maioria das propriedades rurais que se destinam à exploração pecuária no estado de Pernambuco (Freitas et al., 2004).

Nas regiões Norte e Nordeste do Brasil, a produtividade dessa gramínea pode ser considerada alta nas zonas menos secas, já que a mesma tem como centro de origem os vales férteis do continente africano, com precipitações anuais acima de $1000 \mathrm{~mm}$ (Brunken, 1977). Segundo Mello et al.(2002), na Zona da Mata de Pernambuco, o capimelefante apresenta alta taxa de acúmulo de forragem, principalmente nas áreas de altas precipitações e temperaturas, sendo, por isso, uma das principais gramíneas utilizadas na alimentação de ruminantes nessas áreas.

No estado de Pernambuco, o cultivo do capim-elefante (Pennisetum purpureum) nas áreas de maior limitação hídrica do Agreste é realizado predominantemente em baixadas, vazante ou sob irrigação (Cunha et al., 2007). Uma das possibilidades de se obter um genótipo de capim-elefante com maior tolerância ao déficit hídrico é pela hibridação do capim-elefante com o milheto (Pennisetum glaucum), do qual resulta um hibrido interespecífico triplóide, estéril, que pode reunir algumas das características desejáveis das duas espécies (Pereira et al., 2001).

Existem vários mecanismos de tolerância à seca, sendo um deles interpretado como a capacidade das plantas em rebrotar e formar um dossel satisfatório após o término do estresse hídrico. O capim-elefante pode tolerar curtos períodos de seca (Bogdan, 1977; Barreto et al., 2005), embora sua produção seja reduzida (Oliveira et al., 2007) ou nula, quando prevalece o déficit hídrico, apresenta capacidade de recuperação logo após o início das chuvas. No entanto, a literatura não reporta muitas informações sobre esta gramínea quando em estresse hídrico.

Diante do exposto, o objetivo deste trabalho foi avaliar a capacidade de rebrotamento de clones de Pennisetum sp. submetidos a diferentes níveis de tempo de supressão da reposição hídrica, com vistas a seleção dos mais promissores para cultivo nas condições de semiárido do Nordeste brasileiro.

\section{MATERIALE MÉTODOS}

O experimento foi instalado sob condições de casa de vegetação, na sede do 


\section{CLONES DE PENNISETUM SOB ESTRESSE HÍDRICO}

Instituto Agronômico de Pernambuco (IPA), localizado na cidade de Recife $\left(8^{\circ} 04^{\prime}\right.$ latitude Sul e $34^{\circ} 55^{\prime}$ longitude Oeste; 10 metros de altitude). As temperaturas médias mensais máxima e mínima foram 31,5 e $21,0^{\circ} \mathrm{C}$, respectivamente, enquanto que a umidade relativa média do ar foi $73 \%$.

$\mathrm{O}$ experimento foi conduzido em potes com $10 \mathrm{~kg}$ de solo, classificado como Latossolo Vermelho Amarelo distrófico. Os resultados das análises física e química do solo foram o seguinte: $\mathrm{pH}=6,1 ; \mathrm{P}$ disponível= $8 \mathrm{mg} / \mathrm{dm}^{3} ; \mathrm{Ca}=1,50 \mathrm{meq} / 100 \mathrm{~g} ; \mathrm{Mg}=0,60$ $\mathrm{meq} / 100 \mathrm{~g} ; \mathrm{K}=48 \mathrm{mg} / \mathrm{dm}^{3} ; \mathrm{Al}=0,15 \mathrm{meq} / 100$ g; matéria orgânica $=1,41 \%$; areia $=87 \%$; silte $=7 \%$; argila $=6 \%$; densidade aparente $=$ $1,49 \mathrm{~g} / \mathrm{cm}^{3} ;$ densidade real $=2,64 \mathrm{~g} / \mathrm{cm}^{3} ;$ grau de floculação= $100 \%$; umidade a 0,33 atm $=$ $6,50 \%$; umidade a 15 atm $=3,85 \%$ e água disponível $=2,65 \%$. O plantio das estacas para obtenção das mudas foi realizado em casa de vegetação. Para cada clone foram plantadas 35 estacas de, aproximadamente, sete centímetros de comprimento, contendo cada uma, apenas uma gema. Aos 34 dias de plantadas, foram selecionadas as mudas mais vigorosas e uniformes. Oito dias após a transplantação das mudas de Pennisetum, previamente enraizadas em vermiculita, o solo de cada vaso foi adubado com $0,3 \mathrm{~g}$ de $\mathrm{K}_{2} \mathrm{O} ; 0,5 \mathrm{~g}$ de $\mathrm{P}_{2} \mathrm{O}_{5}$ e $1,65 \mathrm{~g}$ de $\mathrm{N}$, tendo como fontes o cloreto de potássio, o superfosfato simples e o sulfato de amônio, respectivamente. Aos 48 dias do experimento, seis dias após o corte de uniformização, foram aplicados 0,67 g/vaso de sulfato de amônio e $0,5 \mathrm{~g} / \mathrm{vaso}$ de uma mistura de micronutrientes composto por zinco, cobre, ferro e manganês.

Os tratamentos foram distribuídos num delineamento inteiramente casualizado em arranjo fatorial, sendo quatro clones de Pennisetum sp. e quatro níveis de estresse hídrico, com três repetições. Três clones foram provenientes dos cultivares de capimelefante Australiano 903-77, Cameroon e Roxo de Botucatu. O quarto clone foi um híbrido de capim-elefante com milheto
(Pennisetum glaucum), obtido pela Instituto Agronômico de Pernambuco-IPA, denominado HV 241. Os quatro níveis de estresse hídrico foram obtidos mediante a suspensão da irrigação por zero, sete, 14 e 21 dias.

$\mathrm{O}$ experimento foi conduzido em três fases. A primeira, com duração de 42 dias, que transcorreu do transplantio de mudas previamente enraizadas até o corte de uniformização, a uma altura de $10 \mathrm{~cm}$. Nesta fase, as perdas de água ocorridas em cada vaso foram repostas a intervalos fixos, a cada 24 horas e depois a intervalos de 48 horas, de forma a manter o solo nos níveis de umidade em torno de $70 \%$ da água disponível. A quantidade de água fornecida $(1000 \mathrm{ml})$ foi superior a capacidade de pote, de forma que, pela diferença entre as quantidades de água fornecida e drenada, foi calculada a evapotranspiração do período. A segunda fase, também com a duração de 42 dias, avaliou a rebrotação subseqüente ao corte de uniformização até o corte após o término do estresse hídrico, sendo nos últimos 21 dias deste período diferenciados os níveis de períodos sem reposição hídrica, e sendo nos primeiros 21 dias a irrigação uniforme em todas as parcelas. Na terceira fase, após a colheita na ocasião do término do estresse hídrico até um período de 14 dias subseqüentes, o solo foi irrigado a cada dois dias, com $1000 \mathrm{ml} / \mathrm{vaso}$, sendo medida a quantidade de água drenada e estimada a evapotranspiração.

A terceira fase (rebrotação após o corte subseqüente ao estresse) iniciou-se após o segundo corte dos tratamentos, tendo os tratamentos submetidos a estresse hídrico na segunda fase recebido um volume de $3000 \mathrm{ml}$, objetivando restaurar a capacidade de campo do solo, volume este fornecido na mesma proporção a todos os níveis por ocasião da instalação do experimento. No que se refere ao tratamento que não recebeu estresse, este permaneceu sob condições de irrigação durante todo o período de condução do experimento.

Durante a segunda fase foram estimadas 
a evapotranspiração (ET), percentagem e produção de matéria seca (MS), eficiência de uso de água (EUA), altura média das plantas, número de perfilhos basais e sintomas de estresse das plantas. Ao final da terceira fase foram estimados números de plantas rebrotadas e de perfilhos basais.

A ET total do período foi calculada pela diferença entre a quantidade de água ofertada e drenada conforme Fernandes Medina e Amaro Filho (1982). A massa seca e a percentagem de MS foram estimadas por meio de corte a $10 \mathrm{~cm}$ do nível do solo, seguido de secagem a $65^{\circ} \mathrm{C}$ até peso constante. A EUA foi estimada pelo quociente entre a evapotranspiração e a produção de MS Para as medições de altura das plantas, foi considerado o comprimento do nível do solo até a extremidade da folha mais alta, esticada verticalmente. Com os dados de altura, foi calculado, por diferença, o crescimento diário da planta. Os dados de crescimento diário foram comparados à idade da planta em cada nível de estresse e clone, pelo coeficiente de determinação $\left(\mathrm{R}^{2}\right)$ e pela equação linear, sendo a idade a variável independente e a altura a variável dependente.

Os sintomas de estresse foram determinados por meio de escala de notas variável entre um e cinco, sendo um o correspondente a ausência de murcha; dois e três para plantas ligeiramente murchas e muito murchas, respectivamente, no período da manhã; quatro para plantas com grande número de folhas com aspecto de palha seca, e cinco para plantas com aspecto de palha seca, conforme escala adaptada por Lira et al. (1989). O número de perfilhos basais e de plantas rebrotadas foram obtidos por contagem nos potes.

Para as variáveis estudadas foram avaliados os efeitos dos fatores clone e nível de estresse e a interação entre esses, conforme Gomes (1981). Os dados obtidos de contagem e notas para sintomatologia de murcha foram submetidos à análise de variância, sendo previamente normalizados pela transformação em raiz quadrada de $\mathrm{x}+1$.
O teste de comparação de médias utilizado foi o Tukey a $5 \%$ de probabilidade. Foram ainda estudadas algumas correlações entre variáveis avaliadas.

\section{RESULTADOSEDISCUSSÃO}

Não houve interação significativa entre clones e nível de estresse hídrico para ETP, produção de MS e EUA (tabela I). Os maiores períodos de supressão da reposição hídrica reduziram mais a ETP do que a produção de MS, tendo a EUA valores de 449 e $591 \mathrm{~g}$ de água/g de MS nos tratamentos 21 dias e sem estresse hídrico, respectivamente (tabela I). O crescimento de plantas forrageiras segue um modelo de curva sigmóide, sendo esta determinada pela disponibilidade de recursos ambientais e variedade. Como o cultivo foi procedido em vasos, provavelmente o

Tabela I. Evapotranspiração potencial (ETP, g/vaso), produção de matéria seca acima de $10 \mathrm{~cm}$ (PMS, g/vaso) e eficiência de uso de água (EUA, g água/g MS) de clones de Pennisetum sp. sob níveis de estresse hídrico. (Potential evapotranspirantion (ETP, g/vessel) dry matter yield (PMS, g/vessel) and water use efficiency (EUA, g water/g DM) of Pennisetum sp. clons under water deficit levels).

\begin{tabular}{lccc}
\hline Clone & EIP & PMS & EUA \\
\hline Australiano & $4862^{\mathrm{ab}}$ & $9,54^{\mathrm{a}}$ & $509^{\mathrm{b}}$ \\
Cameroon & $4407^{\mathrm{b}}$ & $8,16^{\mathrm{ab}}$ & $548^{\mathrm{ab}}$ \\
Híbrido HV 241 & $5167^{\mathrm{a}}$ & $9,30^{\mathrm{a}}$ & $561^{\mathrm{ab}}$ \\
Roxo de Botucatu & $3930^{\mathrm{c}}$ & $6,64^{\mathrm{b}}$ & $635^{\mathrm{a}}$ \\
& & & \\
Estresse hídrico & ETP & PMS & EUA \\
& & & \\
Com irrigação & $6212^{\mathrm{A}}$ & $11,04^{\mathrm{A}}$ & $591^{\mathrm{A}}$ \\
7 d sem irrigação & $4799^{\mathrm{B}}$ & $8,57^{\mathrm{B}}$ & $590^{\mathrm{A}}$ \\
14 d sem irrigação & $4201^{\mathrm{C}}$ & $7,04^{\mathrm{B}}$ & $623^{\mathrm{A}}$ \\
21 d sem irrigação & $3153^{\mathrm{D}}$ & $6,99^{\mathrm{B}}$ & $449^{\mathrm{B}}$ \\
CV (\%) & 9,83 & 19,47 & 19,47 \\
\hline
\end{tabular}

Médias seguidas de letras iguais nas colunas, (minúscula: clone e maiúscula: nível de estresse), não diferem entre si pelo Teste de Tukey $(p>0,05)$. 


\section{CLONES DE PENNISETUM SOB ESTRESSE HÍDRICO}

Tabela II. Notas ${ }^{1}$ atribuidas aos sintomas de murcha ao final do estresse hídrico em clones de Pennisetum $s p$. (Stress symptoms notes at the end the water deficit in Pennisetum sp. clons).

\begin{tabular}{lcccc}
\hline & \multicolumn{5}{c}{ Dias sem irrigação } \\
Clone & 0 & 7 & 14 & 21 \\
\hline Australiano & $1,0^{\mathrm{a}}$ & $3,3^{\mathrm{b}}$ & $3,0^{\mathrm{b}}$ & $5,0^{\mathrm{b}}$ \\
Cameroon & $1,0^{\mathrm{a}}$ & $1,0^{\mathrm{a}}$ & $1,7^{\mathrm{a}}$ & $4,7^{\mathrm{b}}$ \\
HV 241 & $1,0^{\mathrm{a}}$ & $3,0^{\mathrm{b}}$ & $3,7^{\mathrm{b}}$ & $4,7^{\mathrm{b}}$ \\
Roxo de Botucatu & $1,0^{\mathrm{a}}$ & $1,0^{\mathrm{a}}$ & $1,0^{\mathrm{a}}$ & $1,7^{\mathrm{a}}$ \\
CV (\%) & \multicolumn{4}{c}{6,23} \\
\hline
\end{tabular}

${ }^{1}$ Notas variáveis entre 1 e 5 , sendo 1 ausência de sintomas e 5 aspecto de palha seca.

${ }^{a b}$ Médias seguidas de letras iguais na coluna não diferem entre si pelo Teste de Tukey $(p>0,05)$.

avanço no crescimento das plantas limitou o aumento da MS aérea acima de $10 \mathrm{~cm}$ de maneira mais drástica do que da ETP, podendo ter provocado, assim, um consumo de água além do necessário e, consequentemente, redução da EUA. Assim, a maior ETP $(p<0,05)$ do tratamento sem estresse hídrico (tabela I) não teve um correspondente aumento da produção de matéria seca, levando a uma menor EUA deste tratamento, em virtude, provavelmente, do maior tempo de abertura dos estômatos nesse tratamento. Freire (1991), trabalhando com milho (Zea mays), observou que o estresse hídrico promoveu maior EUA do que a ausência de estresse. Por outro lado, Assis e Verona (1991), determinaram que, em sorgo (Sorghum bicolor), a ETP e a EUA aumentaram com a freqüência e a quantidade de água fornecida.

Foi observada uma correlação positiva e significativa $\left(\mathrm{r}=0,8757^{* *}\right)$ entre a MS aérea acima de $10 \mathrm{~cm}$ (g/vaso) e a ET ( $\mathrm{g} \mathrm{H}_{2} \mathrm{O} /$ vaso $)$, não sendo observada mais nenhuma correlação significativa entre a MS aérea acima de $10 \mathrm{~cm}$ e as demais variáveis. A MS aérea acima de $10 \mathrm{~cm}$ do clone Roxo de Botucatu foi inferior $(p<0,05)$ ao do clone Australiano e do híbrido HV 241 (tabela I).
Por outro lado, a MS aérea acima de $10 \mathrm{~cm}$ do tratamento sem estresse foi superior $(p<0,05)$ a todos os tratamentos submetidos a estresse, porém não foram observadas diferenças significativas $(p>0,05)$ entre esses, o que corrobora o maior efeito do estresse hídrico na ET do que na MS aérea acima de $10 \mathrm{~cm}$. Os clones com menor MS aérea acima de $10 \mathrm{~cm}$ apresentaram também menor consumo de água (tabela I), o que provavelmente ocasionou menor déficit hídrico nessas plantas, o que pode ser associado aos menores sintomas de murcha observados (tabela II). Tal fato também foi confirmado pelos teores de matéria seca das plantas (tabela III).

Com relação às notas atribuídas aos sintomas ao final do estresse hídrico (tabela II), ocorreu interação significativa entre o nível de estresse e cultivar. No nível zero, nenhum cultivar apresentou sintoma de estresse. Os clones Australiano e HV 241 apresentaram maiores notas do que Cameroon e Roxo de Botucatu nos dois níveis intermediários, enquanto que, no nível máximo de estresse, o Roxo de Botucatu apresentou menores $(\mathrm{p}<0,05)$ sintomas do que os demais clones. Possivelmente o menor desenvolvimento e ETP expliquem a menor nota de murcha deste clone, podendo

Tabela III. Influência do estresse hídrico na percentagem de matéria seca de clones de Pennisetum $s p$. (Water deficit influence in dry matter content of Pennisetum sp. clons).

\begin{tabular}{lcccc}
\hline & \multicolumn{4}{c}{ Dias sem irrigação } \\
Clone & 0 & 7 & 14 & 21 \\
\hline Australiano & $25,11^{\mathrm{aD}}$ & $53,07^{\mathrm{aB}}$ & $40,29^{\mathrm{bC}}$ & $73,88^{\mathrm{aA}}$ \\
Cameroon & $26,60^{\mathrm{aB}}$ & $24,95^{\mathrm{bB}}$ & $34,32^{\mathrm{bB}}$ & $69,16^{\mathrm{aA}}$ \\
HV 241 & $29,25^{\mathrm{aC}}$ & $46,89^{\mathrm{aB}}$ & $71,52^{\mathrm{aA}}$ & $73,69^{\mathrm{aA}}$ \\
Roxo Botucatu & $20,65^{\mathrm{aB}}$ & $20,32^{\mathrm{bB}}$ & $18,65^{\mathrm{cB}}$ & $30,14^{\mathrm{bB}}$ \\
CV (\%) & \multicolumn{4}{c}{12,43} \\
\hline
\end{tabular}

${ }^{a A}$ Médias seguidas de letras iguais (maiúscula, linhas e minúscula, colunas), não diferem entre si pelo Teste de Tukey $(p>0,05)$. 


\section{SANTOS, LIRA, TABOSA, MELLOE SANTOS}

este fato ser interpretado como um possível mecanismo de tolerância a seca. Segundo Pasin et al.(1991), uma das primeiras reações das plantas às condições de estresse hídrico é o fechamento dos estômatos, o que promove reduções na atividade fotossintética, no crescimento (podendo chegar a paralisar), bem como na taxa respiratória. Este comportamento pode ser considerado benéfico, pois promove maior EUA armazenada no perfil do solo.

O clone Cameroon teve posição intermediária quanto a ET e capacidade de desenvolvimento, característica essa que pode ser visualizada pela emissão de perfilhos basais após estresse hídrico (tabela IV), apresentando menor severidade nos sintomas do que o clone Australiano e o híbrido HV 241, quando submetidos a 14 dias sem irrigação. Lira et al. (1989), cultivaram sorgo em potes, e verificaram que as linhagens com menor altura na fase de plântula, apresentavam menores sintomas quando submetidas a déficit hídrico nesta fase de desenvolvimento. A análise da variância dos dados de percentagem de MS da forragem dos clones ao término do período de estresse hídrico (tabela III) revelou efeitos significativos $(\mathrm{p}<0,05)$ para interação cultivar versus nível de estresse.
No nível zero de estresse, os clones não diferiram $(p>0,05)$ entre si. Sete dias sem irrigação foram suficientes para elevar o teor de MS de $25,11 \%$ para $53,07 \%$ e de $29,25 \%$ para $46,89 \%$, nos clones Australiano e HV 241, respectivamente, não tendo produzido mudanças significativas $(\mathrm{p}>0,05)$ nos dois outros clones. Aos 14 dias sem irrigação, o HV 241 atingiu 71,52\% de MS, sendo significativamente superior $(p<0,05)$ aos demais clones. O cultivar Roxo de Botucatu, aos 21 dias sem irrigação, apresentou $30,14 \%$ de MS, enquanto que o Australiano, Cameroon e Roxo de Botucatu tiveram 73,$88 ; 69,16$ e $73,69 \%$, respectivamente. Como já mencionado, um dos mecanismos de tolerância à seca é o fechamento dos estômatos e redução no crescimento, à medida que aumenta o déficit hídrico. As plantas que não apresentam esse mecanismo, ao não fecharem os estômatos, permanecem realizando trocas gasosas com o ambiente e, portanto, perdendo água e, consequentemente, eleva-se o teor de MS, sendo essas plantas, provavelmente, mais susceptíveis à seca (Aguilar Chavarria, 1985). A percentagem de MS apresentou correlação linear de $0,93(\mathrm{p}<0,01)$ com a nota de sintomatologia da seca. O baixo teor de MS é apontado como um dos fatores limi-

Tabela IV. Número médio de perfilhos basais por vaso de clones de Pennisetum sp. 14 dias após o término do estresse hídrico. (Basal bud number per pot of Pennisetum sp. clons 14 days after the end of water stress).

\begin{tabular}{lccccc}
\hline & \multicolumn{5}{c}{ Nível de estresse hídrico } \\
Clone & $\mathrm{T}_{0}$ & $\mathrm{~T}_{1}$ & $\mathrm{~T}_{2}$ & $\mathrm{~T}_{3}$ & Média \\
\hline Australiano & 7,67 & 5,67 & 4,67 & 2,50 & $5,37^{\mathrm{a}}$ \\
Cameroon & 2,67 & 2,33 & 3,00 & 2,33 & $2,58^{\mathrm{b}}$ \\
HV 241 & 8,00 & 4,00 & 4,00 & 2,00 & $4,73^{\mathrm{ab}}$ \\
Roxo de Botucatu & 1,33 & 0,67 & 0,67 & 0,33 & $0,75^{\mathrm{c}}$ \\
Média & $4,92^{\mathrm{A}}$ & $3,17^{\mathrm{AB}}$ & $3,08^{\mathrm{AB}}$ & $1,70^{\mathrm{B}}$ & \\
\hline
\end{tabular}

$T_{0}=21$ d. I. + (21d.I. + 0d. E.H.); $T_{1}=21$ d. I. + (14d.I. + 7d. E.H.); $T_{2}=21$ d. I. + (7d.I. + 14d. E.H.); $T_{3}=21$ d I. + (0d.I. + 21d. E.H.); I= irrigação; EH= estresse hídrico.

Médias seguidas de letras iguais (maiúscula, linhas e minúscula, colunas), não diferem entre si pelo Teste de Tukey $(p>0,05)$.

Archivos de zootecnia vol. 60, núm. 229, p. 36. 


\section{CLONES DE PENNISETUM SOB ESTRESSE HÍDRICO}

tantes à produção de silagem de gramíneas tropicais (Nussio et al., 2002). Assim, é provável que um estresse hídrico pouco severo antes da colheita eleve um pouco o teor de matéria seca da forragem, facilitando assim a produção de silagem de qualidade.

A capacidade de rebrotação dos clones após o término do estresse hídrico, traduzida pelo número de perfilhos basais avaliados após 14 dias do final do estresse hídrico, ou seja, após a retomada da irrigação, pode ser visualizada na tabela IV. Observou-se, para esta variável, diferenças significativas $(p<0,05)$ para clones e níveis de estresse hídrico e não significativa $(\mathrm{p}>0,05)$ para a interação entre esses fatores.

Nesse contexto, os clones Australiano e HV-241 apresentaram, independentemente do nível de estresse, maior $(\mathrm{p}<0,05)$ número de perfilhos basais do que o clone Roxo de Botucatu, sendo este último, o menos recomendável para a formação de capineiras em áreas sujeitas a estresse hídrico moderado. Além disso, o referido clone também apresentou menor $(\mathrm{p}<0,05)$ PMS (tabela I) que os demais.

Hillesheim e Corsi (1990) afirmam que o número de perfilhos basais do capim-elefante é reduzido drasticamente pelo déficit hídrico. Possivelmente, a redução do número de perfilhos basais do capim-elefante representa um dos principais efeitos do déficit hídrico em relação à baixa sobrevivência de capineiras com essa espécie no semiárido do Nordeste Brasileiro. Pelos resultados do presente estudo, a partir de 21 dias de estresse hídrico, os clones avaliados respondem ao baixo nível de umidade do solo com uma redução no número médio de perfilhos basais.

Os níveis de estresse estudados não levaram nenhum dos quatro clones a morte, já que as plantas rebrotaram em todos os tratamentos, sendo este resultado similar ao obtido por Aguilar Chavarria (1985), também estudando estresse hídrico em capim-elefante. Deste modo, os efeitos principais da deficiência hídrica neste expe- rimento foram a murcha da planta, mensurada pela escala de notas e pelo teor de MS, e a redução do número de perfilhos basais. Apesar de não terem sido realizadas análises da composição química-bromatológica da forragem produzida no presente estudo, estes resultados merecem ser avaliados de maneira conjunta com as possíveis variações no valor nutritivo da forragem, que podem ser decorrentes do estresse sofrido pelas plantas.

A variação da resposta dos clones ao estresse hídrico em relação ao teor de MS, correlacionada à nota de sintomatologia, requer aprofundamento de análise. Prisco (1986) informa que existem basicamente três mecanismos de resistência à seca: fuga ou escape, tolerância com altos níveis de potencial hídrico na planta e tolerância em baixos níveis de potencial hídrico na planta. A fuga à seca consiste na planta completar o seu ciclo vital antes que os tecidos atinjam um déficit hídrico de magnitude que possa afetar seu crescimento normal. Este mecanismo é típico de algumas plantas anuais, podendo ser descartado nessa discussão. A tolerância à seca em altos níveis de potencial hídrico ocorre nas espécies que são capazes de retardar a perda de água. A elevação do teor de MS (tabela III) de três dos quatro clones estudados, indica que eles não foram capazes de retardar a perda de água, à medida que a seca se tornou mais severa. O cultivar Roxo de Botucatu que manteve este potencial, provavelmente foi submetido a menor estresse efetivo, face ao menor desenvolvimento apresentado. $\mathrm{Na}$ tolerância à seca em baixos níveis de potencial hídrico podem-se distinguir dois mecanismos. Os que são responsáveis pela adaptabilidade dos tecidos sem grandes prejuízos ao crescimento, e aqueles que permitem ao protoplasma celular sobreviver à desidratação, possibilitando as plantas recuperarem-se após a remoção do estresse.

Os clones Australiano, Cameroon e HV 241 apresentavam-se desidratados ao final do estresse de 21 dias sem irrigação, entre- 
tanto recuperaram-se após o corte e a finalização do estresse, indicando mais uma vez que, para os clones de Pennisetum sp. avaliados no presente estudo, a capacidade de rebrotação, uma vez restabelecida as condições hídricas favoráveis, pode ser considerada um dos principais mecanismos de tolerância à seca.

Embora para algumas características avaliadas as diferenças observadas não tenham sido significativas, os clones Australiano e HV-241 apresentaram maior ETP, PMS, EUA, sintomatologia de resposta ao estresse hídrico, aumento do teor de MS e do número de perfilhos basais após o término do período de estresse. Consequentemente, uma menor nota de sintomatologia de resposta ao estresse não levou a maior sobrevivência, traduzida por maior número de perfilhos basais após o término do estresse, indicando que o fato do clone ter sofrido maior desidratação do que os demais, não o torna, obrigatoriamente, mais susceptível à seca, conforme apontado na introdução. Sabe-se que vários mecanismos fisiológicos podem interagir nas respostas das plantas ao déficit hídrico, tais como: perda de folhas, associado ou não ao fechamento estomático; o desenvolvimento de órgãos de reserva, fotossíntese do tipo MAC (metabolismo ácido das crassuláceas), redução da área foliar e fechamento

\section{BIBLIOGRAFIA}

Aguilar Chavarria, J.A. 1985. Avaliação da sobrevivência ao estresse hídrico e de outras características morfofisiológicas de sete clones de capim-elefante (Pennisetum purpureum, Schum.) em condições controladas. Dissertação (Mestrado em Zootecnia). Universidade Federal Rural de Pernambuco. Recife. 189 pp.

Assis, F.N. e Verona, L.A.F. 1991. Consumo de água e coeficiente de cultura do sorgo. Pesqui. Agropecu. Bras., 26: 665-670.

Barreto, G.P., Lira, M.A., Santos, M.V.F. e Dubeux Junior, J.C.B. 2005. Produção total de matéria seca e taxa de sobrevivência de clones de estomático (Taiz e Zeiger, 1998). Trabalho conduzido em Pernambuco indicou que a redução do crescimento de plantas do gênero Pennisetum sp. prevalece em condições de déficit hídrico moderado (Barreto et al., 2005).

Vale ressaltar ainda a necessidade de estudos que avaliem essas respostas também em vasos de maior capacidade, com diferentes tipos de solo, maior número de clones, bem como períodos de estresse mais prolongados, visando a seleção de clones mais tolerantes à seca. Embora vários caracteres tenham sido avaliados, a metodologia poderia ser bastante simplificada priorizando a avaliação do número de perfilhos basais após o término do período de estresse hídrico, visto que essa variável sofreu reduções significativas em resposta aos diferentes de tempo de supressão da reposição hídrica.

\section{CONCLUSÕES}

Dentre os clones avaliados, o Australiano e o HV 241 apresentam maior produção de massa seca quando sujeitos a estresse hídrico moderado.

O déficit hídrico elevou o teor de matéria seca dos clones avaliados.

Estudos com maiores períodos de supressão hídrica devem ser realizados, visando avaliar a influência na capacidade de rebrotação dos genótipos avaliados.

capim-elefante (Pennisetum purpureum Schum.) e seus híbridos com o milheto (Pennisetum americanum (L.) Leeke) submetidos a estresse hídrico. Pasturas Tropicales, 27: $27-$ 33.

Bogdan, A.V. 1977. Tropical pastures and folder crops. Longman. New York. 475 pp.

Brunken, J.N. 1977. A systematic study of Pennisetum Sect. Pennisetum (Gramineae). Amer. J. Bot., 64: 161-176.

Cunha, M.V., Lira, M.A., Santos, M.V.F., Dubeux Junior, J.C.B. e Freitas. E.V. 2007. Crescimento inicial de genótipos de Pennisetum sp. sob

Archivos de zootecnia vol. 60, núm. 229, p. 38. 


\section{CLONES DE PENNISETUM SOB ESTRESSE HÍDRICO}

déficit hídrico no Agreste de Pernambuco. Em: XX Reunión Asociación Latinoamericana de Producción Animal (ALPA). Anais... Cusco.

Fernandes Medina, B. e Amaro Filho, J. 1982. Determinação de evapotranspiração real da cultura da cana-de-açúcar na região do brejo paraibano. Agropec. Téc., 3: 11-23.

Freire, F.J. 1991. Efeito do estresse hídrico e da adubação nitrogenada em parâmetros de crescimento do milho (Zea mays L.), teor de nitrogênio e eficiência do uso da água. Dissertação (Mestrado em Agronomia). UFRPE. Recife. $156 \mathrm{pp}$.

Freitas, E.V., Lira, M.A., Dubeux Júnior, J.C.B., Santos, M.V.F., Mello, A.C.L., Tabosa, J.N. e Farias, I. 2004. Características produtivas e qualitativas de clones de capim-elefante (Pennisetum purpureum Schum.) avaliados sob pastejo na zona da mata de Pernambuco. Acta Scient., 26: 251-257.

Gomes, F.P. 1981. Curso de estatística experimental, 9 ed. USP-ESALQ. Piracicaba. 430 pp.

Hillesheim, A. e Corsi, M. 1990. Capim-elefante sob pastejo. I. Fatores que afetam o consumo. Pesqui. Agropecu. Bras., 25: 409-419.

Lehane, L. 1981. How some tropical pastures plants cope with water stress. Rural Res., 111 8-10.

Lira, M.A., Brandão, A.R.M., Tabosa, J.N. e Brito, G.Q. 1989. Estudos preliminares de resistência à seca em genótipos de sorgo forrageiro (Sorghum bicolor (L.) Moench). Rev. Soc. Bras. Zootecn., 18: 1-12.

Mello, A.C.L., Lira, M.A., Dubeux Júnior, J.C.B., Santos, M.V.F. e Freitas, E.V. 2002. Carac- terização e seleção de clones de capim-elefante (Pennisetum purpureum Schum.) na Zona da Mata de Pernambuco. Rev. Bras. Zootecn., 31: 30-42.

Nussio, L.G., Paziani, S.F. e Nussio, C.M.B. 2002. Ensilagem de capins tropicais. Em: Reunião Anual da Sociedade Brasileira de Zootecnia, 39. Recife. Anais... SBZ/UFRPE. Recife. pp. 6099 .

Oliveira, T.N., Santos, M.V.F., Lira, M.A., Mello, A.C.L., Ferreira, R.L.C. e Dubeux Júnior, J.C.B 2007. Métodos de avaliação de disponibilidade de forragem em clones de Pennisetum sp. sob pastejo. Rev. Bras. Ciênc. Agrár., 2: 168-173.

Pasin, N.H., Santos Filho, B.G., Santos, D.S.B. e Mello, V.D.C. 1991. Desempenho de sementes de feijão provenientes de plantas submetidas a déficit hídrico em dois estágios de crescimento. Pesqui. Agropecu. Bras., 26: 183-192.

Pereira, A.V., Valle, C.B., Ferreira, R.P. e Miles, J.W. 2001. Melhoramento de forrageiras tropicais. Em: Nass, L.L., A.C.C. Valois, I.S. Melo, M.C. Valadares Inglis (Ed.). Recursos genéticos e melhoramento de plantas. Fundação MT. Rondonópolis. pp. 449-601.

Prisco, J.T. 1986. Possibilidades de exploração de lavouras xerófilas no semi-árido brasileiro. Pesqui. Agropecu. Bras., 21: 333-342.

Santos, M.C.S., Tabosa, J.N., Dias, F.M., Freitas, E.V. e Lira, M.A. 1994. Comportamento de clones de capim-elefante e de híbridos de capimelefante $\mathrm{x}$ milheto no semi-árido do Nordeste do Brasil. Pesqui. Agropecu. Bras., 29: 1609-1615.

Taiz, L. and Zeiger, E. 1998. Plant physiology, $2^{\text {nd }}$ ed. Sinauer Associates, Inc. Sunderland. 792 pp. 